\title{
Simple multiplex PCR assays to detect common pathogens and associated genes encoding for acquired extended spectrum betalactamases (ESBL) or carbapenemases from surgical site specimens in Vietnam
}

\author{
Ngo Tat Trung', Tran Thi Thu Hien', Tran Thi Thanh Huyen', Dao Thanh Quyen', Mai Thanh Binh², \\ Phan Quoc Hoan ${ }^{1}$, Christian G Meyer ${ }^{3}$, Thirumalaisamy P Velavan ${ }^{3,4}$ and Le Huu Song ${ }^{1,5^{*}}$
}

\begin{abstract}
Surgical site infection (SSI) is common in Vietnamese post-operative patients. It contributes to increased morbidity, mortality, hospitalization time and health care expenditure. Bacterial culture is considered the gold standard procedure to identify SSI pathogens and antibiotic resistant properties; however, it can detect microbes that can readily grow and is time-consuming. We propose optimized multiplex PCR assays to diagnose the most relevant microbes and associated genes encoding for acquired extended spectrum betalactamases (ESBL) or carbapenemases from Vietnamese patients with SSI in a hospital setting in Hanoi.

Methods: Ninety-one patients ( $n=91$ ) were collected in order to identify microbial pathogens and associated genes encoding for acquired extended spectrum betalactamases (ESBL) or carbapenemases by both conventional bacterial culture and in-house multiplex PCR assays.

Result and conclusion: The novel in-house multiplex PCR assays are comparable to the bacterial culture approach in screening for common pathogens causing SSI and for relevant genotypes conferring betalactam/carbapenem resistance for bacteria. This is the first report of Turkey-specific ESBL gene (PER-1) and two Oxacilinase families (Oxa23 and Oxa 58) in Vietnam.
\end{abstract}

Keywords: Surgical site infection, Betalactam/carbapenem resistance, Vietnam

\section{Introduction}

Hospital-acquired bacterial infection is a common problem in post-operative patients and contributes to increased morbidity, mortality, hospitalization time and health care expenditure [1]. Once surgical site infections (SSI) are detected in patients in medical intensive care, antibacterial/antifungal therapy is implemented. However, the efficacy of antimicrobial treatment largely depends on accurate diagnoses of the microbes causing

\footnotetext{
*Correspondence: lehuusong@108-icid.com

'Department of Molecular Biology, Tran Hung Dao Hospital, No 1, Tran Hung Dao Street, Hai Ba Trung Dist, Hanoi, Vietnam

${ }^{5}$ Institute of Clinical Infectious Diseases, Tran Hung Dao Hospital, No 1, Tran Hung Dao Street, Hai Ba Trung Dist, Hanoi, Vietnam

Full list of author information is available at the end of the article
}

SSI. To date, bacterial culture is considered the gold standard procedure to identify pathogens that causing SSI. Staphylococcus aureus, Escherichia coli, Enterococcus spp., Pseudomonas aeruginosa, Enterobacter spp., Candida albicans, Klebsiella pneumonia, Gram-positive anaerobes and Proteus mirabilis are the most common causative pathogens of SSI [2]. However, the classical blood culture procedure has two intrinsic problems: (i) it detects only culturable microbes, thus reducing the chance to identify infectious microorganisms from patients treated with antibiotics; (ii) it takes 24 to 48 hours to achieve first test results of cultures, which hampers accurate treatment and risks the patient's life [3]. Although a broad spectrum of antibiotics is administered 
post-operatively to control SSI, the increasing occurrence of pathogens resistant to a wide spectrum of antibiotics is alarming, such as meticillin-resistant S.aureus (MRSA) or resistance of betalactamases (ESBL)/carbapenemases enterobacteriacea $[4,5]$. So far, more than one thousand betalactamase genes encoding either ESBL or the carbapenemase phenotype have been recognized [6] (http://www.lahey.org/studies/webt.asp). The distribution of these clinical phenotypes differs greatly between geographical settings and displays distinct local patterns. If conventional bacterial cultures fail to generate colonies, antibiotic resistance profiles cannot be generated.

Vietnam is a tropical country where the risk of infectious diseases remains high. The incidence of SSI detected by bacterial culture reaches up to 33\% [7]. Only a few sporadic reports on SSI-related pathogens and no conclusive data on resistance phenotypes are available from Vietnam. Systematic statistics on the betalactamase genotypes that cause epidemics are lacking. Own studies indicate that Staphylococcus epidermidis, E. coli, Pseudomonas auriginosa, Streptococcus spp., Klebsiella pneumonia, Enterobacter spp., Staphylococcus aureus and Candida spp. are the most culturable SSI-causative pathogens and we suspect resistance patterns in the genes encoding VEB, CTX-M of ESBL group or NDM-1 of carbapenemase. Here, we introduce simple multiplex PCR assays that provide rapid diagnostic applications and supplement classical bacterial cultures. Furthermore, our multiplexed PCR approach enables to estimate the prevalence of ESBL and/or carbapenem resistance phenotypes in Vietnamese patients with deep incisional SSI.

\section{Material and methods Sampling}

Eligible study participants were patients hospitalized at Tran Hung Dao Hospital, Hanoi, Vietnam, between
February 2012 and December 2012 with at least one of the following conditions: (1) purulent incisional drainage from deep layers of soft tissue within 30 days of the operation or within 1 year of the operation if a prosthesis was implanted; (2) local signs and symptoms of pain or tenderness, swelling, and erythema, with the incision opened by the surgeon or confirmed by the attending surgeon or physician. Patients provided informed consent and the study was approved by the ethics committee of Tran Hung Dao Hospital. Exudates from deep incisional surgical infection sites (aspirate beneath the incision area) were collected using sterile syringes and, in parallel, subjected to bacterial culture or stored at $-80^{\circ} \mathrm{C}$ for molecular diagnostics. Ninety-one patients $(\mathrm{n}=91)$ were enrolled in the study.

\section{Bacterial isolates}

For optimization of multiplex PCR assays, well-characterized, both biochemically and molecularly, colonies of Candida albicans, Acinetobacter baumanni, Pseudomonas aeruginosa, Klebsiella pneumoniae, Staphylococcus aureus, Streptococcus pneumoniae, Staphylococcus epidermidis and Escherichia coli were isolated from clinical isolates at the Department of Clinical Microbiology, Tran Hung Dao Hospital, Hanoi, Vietnam.

\section{Bacterial culture}

For the establishment of bacterial cultures we followed the Manual of Clinical Microbiology [8]. Briefly, $500 \mu \mathrm{l}$ aliquots of the purulent incisional drainage from individual biopsies was vortexed well in $800 \mu$ l sterilized phosphate buffer saline (PBS). The resulting suspension was streaked onto 4 solid media: sheep blood agar, MacConkey agar, chocolate agar and buffered charcoal-yeast extract agar (BCYE) under aerobic conditions at $37^{\circ} \mathrm{C}$ for 7 days. Colonial growth was confirmed by medical microbiologists at the Department of Clinical Microbiology, Tran Hung Dao Hospital, Hanoi, Vietnam.

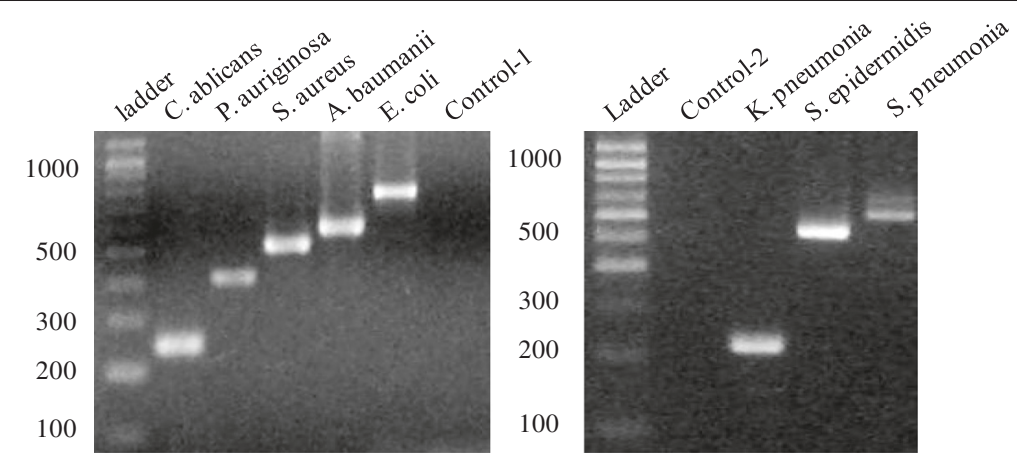

Figure 1 Multiplex PCR assays for screening of SSI associated microorganisms. Left panel MicroSHPT@5leX containing primers specific for Candida albicans, Acinetobacter baumanni, Pseudomonas aeruginosa, Staphylococcus aureus, Escherichia coli, whereas MicroSHPT@3leX amplify target genes specific for Klebsiella pneumonia Streptococcus pneumonia, Staphylococcus epidermidis. Control-1, control-2 are human whole blood genomics DNA. 
Design of genus specific primers for multiplex PCR assays to detect microorganisms

To screen for the 8 microbes indicated above, two multiplex PCR assays (MicroSHPT@5leX and MicroSHPT@3leX) were designed such that neighboring amplicons differed by 50 to $100 \mathrm{bps}$ in size, helping to resolve PCR fragments into visible bands in agarose gel electrophoresis (Figure 1).
Design of family specific primers for multiplex PCR assays to screen for relevant ESBL and carbapenemase encoding genes

To screen for genes of important ESBL and carbapenemases, five multiplex PCR assays, SHPT@ESBL-1 (SHV, TEM, CTX-M), SHPT@ESBL-2(VEB, GES, PER), SHPT@Carba-1(NDM, SPM, VIM), SHPT@Carba-2(IMP, AIM, KPC/BIC, DIM), SHPT@Carba-3(Oxa23 like group,

Table 1 Primer sequence used for screening of genes coding ESBL or carbapenemase

\begin{tabular}{|c|c|c|c|}
\hline \multicolumn{3}{|c|}{ SHPT@ESBL-1(SHV, TEM, CTX-M) } & \multirow{2}{*}{$\begin{array}{l}\text { Final concentration ( } \mathrm{mM}) \\
0.4\end{array}$} \\
\hline 590 & SHPT108@CTX-M-U1-F & ATGTGCAGYACCAGTAARGTKATGGC & \\
\hline & SHPT108@CTX-M-U2-R & GGTRAARTARGTSACCAGAAYCAGCGG & 0.4 \\
\hline \multirow[t]{2}{*}{422} & SHPT108@TEM-U-F & TCGCCGCATACACTATTCTCAGAATGAC & 0.08 \\
\hline & SHPT108@TEM-U-R & CAGCAATAAACCAGCCAGCCGGAAG & 0.08 \\
\hline \multirow[t]{2}{*}{739} & SHPT108@TR-SHV-F & TGTATTATCTC(C/T)CTGTTAGCC(A/G)CCCTG & 0.48 \\
\hline & SHPT108@TR-SHV-R & GCTCTGCTTTGTTATTCGGGCCAAGC & 0.48 \\
\hline \multicolumn{4}{|c|}{ SHPT@ESBL-2(VEB, GES, PER) } \\
\hline \multirow[t]{2}{*}{391} & SHPT108@TR-VIM-F & GATGGTGTITGGTCGCATATCGCAAC & 0.08 \\
\hline & SHPT108@TR-VIM-R & CATCGCTGTTGGGGTTGCCCAATTIT & 0.08 \\
\hline \multirow[t]{2}{*}{604} & SHPT108@TR-SPM-F & CTGGCAGGGATCGCTCACTC & 0.08 \\
\hline & SHPT108@TR-SPM-R & GGTTTCCGATCAGCCACCTCTCA & 0.08 \\
\hline \multirow[t]{2}{*}{731} & SHPT108@NDM-1-F primer & CAGTGTGGGGGCCTGACGAT & 0.08 \\
\hline & SHPT108@NDM-1-R primer & CTGAGCA ACC TGC GCA ATR ATA GCT T & 0.08 \\
\hline \multicolumn{4}{|c|}{ SHPT@Carba-1(NDM, SPM, VIM) } \\
\hline \multirow[t]{2}{*}{320} & SHPT108@TR-VIM-F & GATGGTGTTTGGTCGCATATCGCAAC & 0.04 \\
\hline & SHPT108@TR-VIM-R & CGAATGCGCAGCACCAGGATAGAA & 0.04 \\
\hline \multirow[t]{2}{*}{291} & SHPT108@TR-SPM-F & CGTTTGAAAATCTGGGTACGCAAACG & 0.04 \\
\hline & SHPT108@TR-SPM-R & GTTCAAATCAAAAACATTATCCGCTGGAACAG & 0.04 \\
\hline \multirow[t]{2}{*}{200} & SHPT108@NDM-1-F primer & CGAAAGTCAGGCTGTGTTGCGC & 0.08 \\
\hline & SHPT108@NDM-1-R primer & GACCGCCCAGATCCTCAACTG & 0.08 \\
\hline \multicolumn{4}{|c|}{ SHPT@Carba-2(IMP, AIM, KPC/BIC, DIM) } \\
\hline \multirow[t]{2}{*}{710} & SHPT108@Tr-DIM-F & TATTCAGCTTGTCTTCGCTTGCTAACG & 0.08 \\
\hline & SHPT108@Tr-DIM-R & GTTAGCGTTCGGCTGGATTGATTG & 0.08 \\
\hline \multirow[t]{2}{*}{412} & TR-KPC-BIC-F & GCTTTCT(T/G)GCTG(C/G)CGC(T/C)GTGCT & 0.2 \\
\hline & TR-KPC-BIC-R & AGCCAATCAAC(A/C)A(A/G)CTGCTG(C/A)CGC & 0.2 \\
\hline \multirow[t]{2}{*}{326} & SHPT108@AIM-F & CCCTGAAGGTGTACGGAAACAC & 0.04 \\
\hline & SHPT108@AIM-R & GGGTTCGGCCACCTCGAATTG & 0.04 \\
\hline \multirow[t]{2}{*}{204} & Tr-IMP-F & AC(G/A)GG(C/G/T)GGAATAGAGTGGCTTAA(T/C)TCTC & 0.02 \\
\hline & TR-IMP-R & TTCAGG(C/T)A(A/G)CCAAACYACTASGTTATCT & 0.02 \\
\hline \multicolumn{4}{|c|}{ SHPT@Carba-3(Oxa23 like group, Oxa48 like group, Oxa58 like group) } \\
\hline \multirow[t]{2}{*}{599} & SHPT108@Oxa-58-F & CCCCTCTGCGCTCTACATACAACATC & 0.08 \\
\hline & SHPT108@Oxa-58-R & AAGTATTGGGGCTTGTGCTGAGCATAG & 0.08 \\
\hline \multirow[t]{2}{*}{482} & Tr-OXA-G23-F2 & AGAATATGT(G/C)CC(A/T)GC(C/A)TC(T/A)ACATTTAA(A/G)ATG & 0.2 \\
\hline & TR-OXA-G23-R2 & $\mathrm{CCCA}(\mathrm{G} / \mathrm{A}) \mathrm{CC}(\mathrm{G} / \mathrm{T}) \mathrm{GT}(\mathrm{C} / \mathrm{T}) \mathrm{AACCA}(\mathrm{G} / \mathrm{A}) \mathrm{CC}$ & 0.2 \\
\hline \multirow[t]{2}{*}{$286 b$} & Tr-Oxa-G48-F2 & CACCAAGTCTITAAGTGGGATGGACA & 0.08 \\
\hline & Tr-Oxa-G48-R2 & CCGATACGTGTAACTTATTGTGATACAGCTT & 0.08 \\
\hline
\end{tabular}


Table 2 Target genes and primer sequences used for screeningof infectious microorganisms

\begin{tabular}{|c|c|c|c|c|}
\hline Pathogens & Accession number & Forward/Reverse Primer (5' to $3^{\prime}$ ) & Size (bp) & Final concentration $(\mathrm{mM})$ \\
\hline \multirow[t]{2}{*}{ C. albicans } & Z48339 & GTGGGTGGTAAATTCCATCTAAAGCTA & 243 & 0.2 \\
\hline & & CCGTGCCACATTCCTCCGC & & 0.2 \\
\hline \multirow[t]{2}{*}{ P. aeruginosa } & AF116258 & CCCGAATGTCGGCATCATTCTC & 411 & 0.2 \\
\hline & & CGGTAGACCTCGCGCTTGAA & & 0.2 \\
\hline \multirow[t]{2}{*}{ S. aureus } & STAAROA & AAGGGCGAAATAGAAGTGCCGG & 515 & 0.2 \\
\hline & & ATGGTCGGTTCCTTAGAAAACAAACTTG & & .02 \\
\hline \multirow[t]{2}{*}{ A. baumannii } & JX470958 & TTGGGGCCTTTGAGGCTTTAGTG & 599 & 0.2 \\
\hline & & TGGTGCAACAAACTCCCATGGT & & 0.2 \\
\hline \multirow[t]{2}{*}{ E. coli } & S-uidA & GTCGCGAGTGAAGATCCCTITC & 773 & 0.2 \\
\hline & & GCATTAATGGACTGGATTGGGGC & & 0.2 \\
\hline \multirow[t]{2}{*}{ K. pneumoniae } & Kp-aldA & CCTTGTCTITAAACGCGCGC & 332 & 0.2 \\
\hline & & TITTCGCCGCAGCGG & & 0.2 \\
\hline \multirow[t]{2}{*}{ S. epidermidis } & AF298800 & CGGTATCTTAGTTGTATCTGCTGC & 637 & 0.2 \\
\hline & & AGATAATACGTATACTTCAGCTITGAATTTTGTG & & 0.2 \\
\hline \multirow[t]{2}{*}{ S. pneumoniae } & Sp LytA & CAACCGTACAGAATGAAGCGGATTAT & 701 & 0.2 \\
\hline & & GTCCTTGTACTTGACCCAGCCT & & 0.2 \\
\hline
\end{tabular}

Oxa48 like group, Oxa58 like group) were designed. The sizes of neighboring PCR amplicons differed by 50 to 100 bps, again supporting resolution of PCR fragments on agarose gels (Figure 1). Except for the SHPT@Carba-3 (Oxa group) multiplex PCR, all primers target the conserved domain of ESBL gene families (SHV, TEM, CTX-M, VEB, GES, PER)or carbapenemase gene families (NDM, SPM, VIM, IMP, AIM, KPC/BIC, DIM). Primer pairs utilized for screening of genes coding ESBL or carbapenemase are listed in Table 1. The reference strains harboring genetic materials encoding for Oxa48, VIM, SIM, SPM, AIM, IMP, KPC, BIC, DIM were kindly provided by Laurent Poirel [9].

\section{DNA extraction and multiplex PCR}

An aliquot of the purulent incisional drainage from individual biopsies was streaked onto solid medium for total bacterial culturing; the remnant was immersed into $300 \mu \mathrm{l}$ universal lysis solution (200 mM NaOH, 1\% SDS) and heated for 5 minutes at $95^{\circ} \mathrm{C}$. An equal volume of $1 \mathrm{M}$ Tris- $\mathrm{HCl}$ was added to neutralize the $\mathrm{pH}$ to 7.5 . This aqueous solution was subjected to standard phenol/chloroform/isoamyl alcohol extraction [10]. Briefly, $600 \mu \mathrm{l}$ of aqueous solutionwere transferred into a $1.5 \mathrm{ml}$ Eppendorf tube, an equal volume of phenol/chloroform/ isoamyl (24/25/1) was added and vortexed for 5 minutes and centrifuged at 13,000 g. The upper aqueous phase was then transferred to an Eppendorf tube and an equal volume of isopropanol was added. After thorough mixing, the solution was centrifuged at $16,000 \mathrm{~g}$ for $30 \mathrm{mi}-$ nutes to pellet the DNA. Precipitated DNA was washed twice with $70 \%$ ethanol and reconstituted in $150 \mu \mathrm{l}$ TE (25 mM Tris-base pH8.0, $1 \mathrm{mM}$ EDTA) and used as template for multiplex PCR in $25 \mu \mathrm{l}$ reactions containing $10 \mathrm{mM}$ Tris- $\mathrm{HCl} \mathrm{pH} 8.3 / 50 \mathrm{mM} \mathrm{KCl} / 1.5 \mathrm{mM} \mathrm{Mg}{ }^{+}$, $250 \mu \mathrm{M}$ of each deoxynucleotide triphosphate and variable amounts of individual primers (Tables 1, and 2). Thermal cycling comprised initial denaturation at $95^{\circ} \mathrm{C}$ for 4 minutes, 35 cycles of $94^{\circ} \mathrm{C}$ for 25 seconds, $58^{\circ} \mathrm{C}$ for 45 seconds and $72^{\circ} \mathrm{C}$ for one minute. In case the multiplex PCR assays, the amplified products were purified and subjected to PCR sequencing. The resulting sequences reading were compared to gene sequences as

Table 3 Positive rates of Vietnamese SSI specimens detected by multiplex PCR assays and bacterial cultures

\begin{tabular}{lll}
\hline Bacterial strain & Bacterial culture & Multiplex PCR \\
\hline Candida albicans & 0 & 0 \\
Acinetobacter baumanni & 4 & 12 \\
Pseudomonas aeruginosa & 9 & 18 \\
Klebsiella pneumonia & 5 & 4 \\
Staphylococcus aureus & 12 & 13 \\
Streptococcus pneumonia & 0 & 0 \\
Staphylococcus epidermidis & 3 & 3 \\
Escherichia coli & 6 & 10 \\
Citrobacter sp & 1 & 0 \\
Total (positive) & $\mathbf{4 0}$ & $\mathbf{6 0}$ \\
Total patient samples & $\mathbf{9 1}$ & $\mathbf{9 1}$ \\
\hline
\end{tabular}


described in the NCBI database (Blast search http:// blast.ncbi.nlm.nih.gov/Blast.cgi).

\section{Results}

Multiplex PCR assays enhance positive rate of SSI

After optimizing amplifying conditions for MicroSHPT@5leX and MicroSHPT@3leX (Figure 1, Table 2), the multiplex PCR assays were applied to screen for infected pathogens from 91 SSIs. Fourty specimens tested positive for colonies of at least one strain and 60 out of the 91 SSI specimens were confirmed as bacterial infections by multiplex PCR assays (Table 3). With the exception of Citrobacter spp., all positive colonies of any SSIs specimen were confirmed by either MicroSHPT@5leX or MicroSHPT@3leX reactions. Four samples were negative by bacterial culture but positive by multiplex PCR assays. Bacterial culture detected fewer infected samples than did the multiplex PCR assays. This clearly demonstrates that our multiplex PCR assays are more sensitive and accurate in detecting SSI than bacterial cultures.

\section{Genotypic prevalence of betalactamases detected from SSI specimens}

After optimizing the amplification conditions for SHPT@ESBL-1, SHPT@ESBL-2, SHPT@Carba-1, SHPT@ Carba-2, SHPT@Carba-3 assays (Figure 2), the five multiplex PCR reactions were used to screen for potential ESBL or cabapenemase genotypes from all 60 PCR-positive SSI samples (Table 3). The screening revealed SHV as the most common ESBL gene family; SHV genotypes existed in 10 (16.6\%) of 60 SSI PCR-positive samples. Other ESBL gene families such as CTX-M or TEM were also detected in a considerable number of biopsies (Table 3). Especially, five of 60 SSI PCR-positive samples (NKSM9, NKSM11, NKSM28, NKSM33, NKSM40) were positive for carbapenemase NDM-1; and also had either SHV or CTX-M or TEM. One
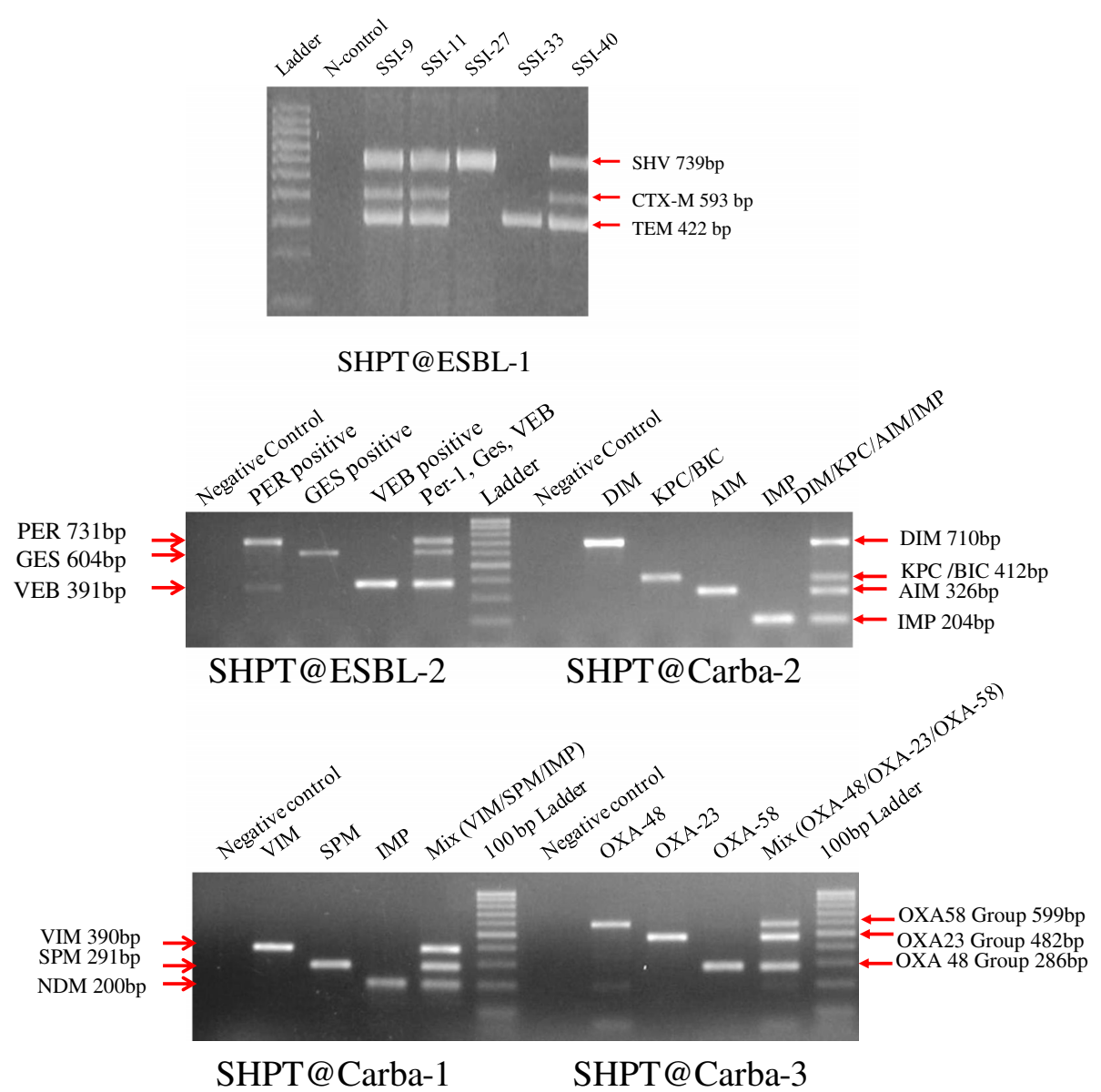

Figure 2 Multiplex PCR assays for screening of acquired ESBL and carbapenemase encoding genes from SSI specimens. Upper left panel is SHPT@ESBL-1 assay to detect SHV, TEM, CTX-M genes; upper right panel is SHPT@ESBL-2 assay to detect VEB, GES, PER genes; lower left panel is SHPT@Carba-1 to screen for NDM, SPM, VIM and SHPT@Carba-2 to screen for IMP, AIM, KPC/BIC, DIM; lower right panel is SHPT@Carba-3 to screen for Oxa23 like group, Oxa48 like group, Oxa58 like group; control samples are human whole blood genomics DNA. 
Table 4 Genotypic prevalence of betalactamases detected from SSI specimens

\begin{tabular}{|c|c|c|c|c|c|c|c|c|c|c|c|}
\hline \multirow[b]{2}{*}{ SSI ID } & \multicolumn{2}{|c|}{ Bacterial strain bearing the betalactamse genes } & \multicolumn{9}{|c|}{ Detected betalactamse genes by multiplex PCR assays } \\
\hline & Multiplex CR & Bacterial culture & NDM 1 & SHV & CTX-M & TEM & $0 \times 23$ & Per-1 & GIM-1 & VEB & OXA-58 \\
\hline NKSM9 & $\begin{array}{l}\text { E. coli, K. pneumonia, } \\
\text { A. baumanii }\end{array}$ & E. coli & Positive & Positive & Positive & Positive & & & & & \\
\hline NKSM11 & E coli & E coli & Positive & Positive & Positive & Positive & & & & & \\
\hline NKSM28 & A. baumanii & Negative & Positive & Positive & Positive & Positive & & & Positive & Positive & Positive \\
\hline NKSM33 & $\begin{array}{l}\text { E. coli, K. pneumonia, A. } \\
\text { baumanii, P. aurigninosa }\end{array}$ & $\begin{array}{l}\text { E. coli, } P \text {. } \\
\text { aurigninosa }\end{array}$ & Positive & Positive & Positive & Positive & & & & & \\
\hline NKSM40 & K. Pneumonia & K. Pneumonia & Positive & Positive & Positive & Positive & & & & & \\
\hline NKSM49 & P. aurigninosa & P. aurigninosa & & Positive & & & & & & & \\
\hline NKSM53 & P. aurigninosa, S. aureus, Ecoli & Ecoli & & Positive & & Positive & & & & & \\
\hline NKSM54 & A. baumanii & Negative & & Positive & & & & & & & \\
\hline NKSM55 & P. aurigninosa & P. aurigninosa & & Positive & & & & & & & \\
\hline NKSM91 & A. baumanii & A. baumanii & & & & & Positive & Positive & & & Positive \\
\hline Total n(\%) & & & $5(5)$ & 9(9) & $5(5)$ & $6(6)$ & $1(1)$ & $1(1)$ & $1(1)$ & & $2(2)$ \\
\hline
\end{tabular}

sample (SSI28) had Turkey-specific ESBL gene (PER-1) and oxacilinase gene families (Oxa23 and Oxa 58).

\section{Discussion}

Hospital acquired bacterial infections after surgical interventions and antibiotic resistances are a major public health threat. Here, we report two optimized multiplex PCR assays that can detect genetics materials of eight most common microbial pathogens, namely Candida albicans, Acinetobacter baumanni, Pseudomonas aeruginosa, Klebsiella pneumoniae, Staphylococcus aureus, Streptococcus pneumoniae, Staphylococcus epidermidis and Escherichia coli. The assays have detection limits ranging from 10 to $50 \mathrm{CFU} / \mathrm{ml}$ human blood (unpublished data). The bacterial identification capability of our multiplex PCR is comparable to that of the conventional blood culture approach, especially in some patient biopsies. PCR was more sensitive than blood cultures.

Antibiotic resistance related to nosocomial infections is of increasing concern and requires the development of suitable tracking tools. Clinical experience in our institution has led us to suspect the existence of microorganisms with extended-spectrum betalactamase or carbapenemase capability. However, under antibiotic pressure in the course of surgical prophylaxis some bacteria cannot proliferate. To overcome this challenge, we have optimized relevant multiplex PCR assays to track for Vietnam-specific ESBL such as VEB-1 [11], blaCTX-M-15, blaCTX-M-27 genes [12] or other dangerous ESBL genes such as NDM-1, OXA48, GIM-1. Our data reveal that, despite the lack of colony growth from patient samples NKSM28, NKSM54 (Table 4), our PCR assays could detect the bacterial genetic material and relevant betalactamase encoding genes (NDM-1, SHV, CTX-M, GIM-1, VEB, OXA-58 in patient sample NKSM28, or SHV in NKSM54 - Table 4).
Although PCR based assays are much sensitive and can detect microbes causing SSI, conventional blood culture procedures should not be ignored for obvious reasons. In addition, PCR based assays alone will not allow to get a comprehensive profile SSI related pathogens or SSI associated antibiotic resistance profiles. Therefore, both methodologies should be considered which shall provide vital information on appropriate administration of drugs during SSI.

\section{Conclusion}

In summary, we developed simple optimized multiplex PCR assays that clearly enhance the positive diagnostic rate of deep incisonal SSI compared to the conventional bacterial culture approach. Our multiplex PCR assays also quickly detected important associated genotypes that confer betalactam resistance for bacteria. Also this is the first report of Turkey-specific ESBL gene (PER-1) and two Oxacllinase families (Oxa23 and Oxa 58) in Vietnam.

\section{Competing interests}

The authors declare that they have no competing interests.

Authors' contributions

NTT and LHS designed and supervised the studies and prepared the manuscript, PQH, MTB provided clinical samples, TTH, TTH and DTQ conducted the experiments, TPV and CGM involved in study design, analyzed the data and proofread the manuscript. All authors read and approved the final manuscript.

\section{Acknowledgements}

We would like to acknowledge the funding from KC-10.43/11-15 for this study.

\section{Author details}

'Department of Molecular Biology, Tran Hung Dao Hospital, No 1, Tran Hung Dao Street, Hai Ba Trung Dist, Hanoi, Vietnam. ²Department of Gastroenterology, Tran Hung Dao Hospital, No 1, Tran Hung Dao Street, Hai Ba Trung Dist, Hanoi, Vietnam. ${ }^{3}$ Institute of Tropical Medicine, University of 
Tuebingen, Tuebingen, Germany. ${ }^{4}$ Fondation Congolaise pour la Recherche Medicale, Brazzaville, Republic of Congo. ${ }^{5}$ Institute of Clinical Infectious

Diseases, Tran Hung Dao Hospital, No 1, Tran Hung Dao Street, Hai Ba Trung Dist, Hanoi, Vietnam.

Received: 8 November 2014 Accepted: 18 March 2015

Published online: 12 April 2015

\section{References}

1. Owens CD, Stoessel K. Surgical site infections: epidemiology, microbiology and prevention. J Hosp Infect. 2008;70 Suppl 2:3-10.

2. Qadan M, Cheadle WG. Common microbial pathogens in surgical practice. Surg Clin North Am. 2009;89(2):295-310. vii.

3. Mancini N, Carletti S, Ghidoli N, Cichero P, Burioni R, Clementi M. The era of molecular and other non-culture-based methods in diagnosis of sepsis. Clin Microbiol Rev. 2010;23(1):235-51.

4. Cao V, Lambert T, Nhu DQ, Loan HK, Hoang NK, Arlet G, et al. Distribution of extended-spectrum beta-lactamases in clinical isolates of Enterobacteriaceae in Vietnam. Antimicrob Agents Chemother. 2002;46(12):3739-43.

5. Nordmann P, Naas T, Poirel L. Global spread of Carbapenemase-producing Enterobacteriaceae. Emerg Infect Dis. 2011;17(10):1791-8.

6. Jacoby GA, Munoz-Price LS. The new beta-lactamases. N Engl J Med. 2005;352(4):380-91.

7. Nguyen D, MacLeod WB, Phung DC, Cong QT, Nguy VH, Van NH, et al Incidence and predictors of surgical-site infections in Vietnam. Infect Control Hosp Epidemiol. 2001;22(8):485-92.

8. Murray PR, Baron EJ, Pfaller MA, Tenover FC, Yolken RH. Manual of clinical microbiology. Washington. D.C: ASM Press; 1999.

9. Poirel L, Walsh TR, Cuvillier V, Nordmann P. Multiplex PCR for detection of acquired carbapenemase genes. Diagn Microbiol Infect Dis. 2011;70(1):119-23.

10. Ausubel FBR, Kingston R, Moore D, Seidman JG, Smith J, Struhl K. Short protocols in molecular biology. 3rd ed. 1995. p. 2-3.

11. Naas T, Benaoudia F, Massuard S, Nordmann P. Integron-located VEB-1 extended-spectrum beta-lactamase gene in a Proteus mirabilis clinical isolate from Vietnam. J Antimicrob Chemother. 2000;46(5):703-11.

12. Trang NH, Nga TV, Campbell JI, Hiep NT, Farrar J, Baker S, et al. The characterization of ESBL genes in Escherichia coli and Klebsiella pneumoniae causing nosocomial infections in Vietnam. J Infect Dev Ctries. 2013;7(12):922-8.

\section{Submit your next manuscript to BioMed Central and take full advantage of:}

- Convenient online submission

- Thorough peer review

- No space constraints or color figure charges

- Immediate publication on acceptance

- Inclusion in PubMed, CAS, Scopus and Google Scholar

- Research which is freely available for redistribution 